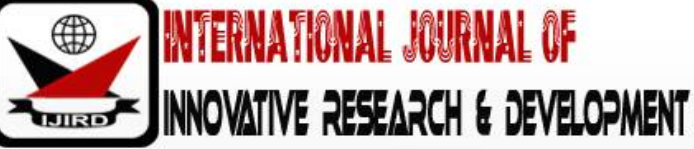

ISSN 2278 - 0211 (Online)

\section{Awareness and Utilization of Mother Child Health Booklet by Health Workers and Mothers in Western Kenya}

Dr. Jane Naliaka Situma
Lecturer, Department of Nutritional Sciences,
Masinde Muliro University of Science and Technology, Kenya
Brenda Ahoya
Postgraduate Student, Department of Nutritional Sciences,
Masinde Muliro University of Science and Technology, Kenya
Rhoda Loreen Musungu
Postgraduate Student, Department of Nutritional Sciences
Masinde Muliro University of Science and Technology, Kenya

\begin{abstract}
:
The mother child health booklet (MCHB) captures information from pregnancy until the child is five years of age. It has been adopted by many countries due to its many advantages. The aim of this study was to establish the level of awareness and utilization of the MCHB by the health workers and mothers in Western region of Kenya. The objectives of the study were to: establish level of awareness among health workers and mothers and utilization of the MCHB by health workers and mothers. Purposive sampling strategy and systematic random sampling strategy were used. A total of 962 clients were sampled. Structured questionnaires, content observation checklist and key informants guides were used to collect data. Data was analyzed using SPSS statistical package version 20. Data was presented in modes, mean and percentages. The results indicate that the level of utilization of the MCHB is different depending on the indicator. Immunization was the most complete at $95 \%$. Among the counties completeness was between 91.5\% (216) in Busia and 98.8\% (250) in Vihiga,p value 0.001. Under health worker consultation, 62.7\% (614) was blank/ never filled at all. Among counties, 62.6\% (154), 48.6\% (123), 64.8\%7 (158), $75.8 \%$ (179) had not been filled in Kakamega, Vihiga, Bungoma and Busia respectively. The age of the mothers was found to influence the number of ANC visits with the highest number of ANC visits being among the older cohort, $\mathrm{p}$ value 0.012 . Overall, $93 \%$ delivered in a health facility with $6.8 \%$ home deliveries. The results of this study will be key in informing decisions about appropriate use of MCHB. Information obtained from this study will also be significant to the national reviewing of the booklet
\end{abstract}

Keywords: Awareness, utilization, mother child booklet, health worker, mother

\section{Introduction}

Mother Child Health Booklet (MCHB) is a single document, documenting both Antenatal and Child Welfare services and belongs to the expectant mother as a client of the health services (JICA, 2012). The first part contains the mother's full antenatal and post-natal profile while the second part contains the child's details on immunizations and other services delivered before five years of age (HMI, 2008), ensuring continuity of maternal, neonatal and child health care (Nakamura, 2010). It is therefore, used to monitor the pregnant woman, her child, their service uptake, survey the provision of health services, promote health education and communication and also provides a reference in case of referrals (JICA, 2012; Kenshiro, 2012). As a result, the MCHB has therefore become an indispensable tool (Imamura, 2012).

Globally, the MCHB is being implemented in both developed and developing countries, with the support of United Nations (UN), JICA and other Non-Governmental Organizations (NGOs) operating in the respective countries (Nakamura, 2010).Countries have periodically revised and updated the MCHB to meet the evolving needs of both providers and users (Isaranurug, 2009; Nakamura, 2010). The Kenyan handbook was revised and updated in 2013 and 2016 to meet the needs of its stakeholders.

Kenya has a very informative MCHB that has all the information the parents need including what and how the mother's or partner can help. It is distributed free of charge during the first ANC visit and every mother/ child pair should be issued with one (MOH, 2013).The MCHB is client held and therefore should enable families' greater ability to track their own health and develop preventive strategies. Furthermore, pregnant women/mothers can use the MCHB as a selflearning tool to improve their knowledge of perinatal care and childcare thus empowering them to participate in taking decision related to their own health and the health of their children (Kenshiro, 2012). 


\section{Literature Review}

Regionally, a study done in Nigeria showed that the level of awareness of the MCHB was significantly influenced by maternal age, education status and parity. Of those who had heard about growth charts, only $55.8 \%$ had them. (Fagbule et al., 1990). A study in Lesotho showed that the mothers who understand the road to health card used the clinics more for growth monitoring (Asuzu, 1991). Reasons given for mothers not carrying the MCHB were that; they thought that they were not needed for such visits, the child's mother was not at home, the child was visiting a relative, it was lost, burnt or never had one (Sistiarani et al., 2016).Therefore, this study seeks to establish whether these are the same reasons that affect utilization of MCHB by mothers and health workers or there are other unique reasons that affect utilization of mother child booklet in western region.

On utilization, a study by Sistiarani et al., 2015 showed that mothers utilization of the MCHB increased by $10 \%$ after being taught how to use the book while their knowledge increased by $20 \%$, while Opondo (2015), found a strong association between utilization and level of education for caregivers. Fagbule et al., (1990) also found that of the $55.8 \%$ of mothers who had the booklet, $96.8 \%$ used it regularly. What is not clear in this study is whether level of education of caregivers in western region affects the awareness and utilization of the booklet hence the need for the study.

In Indonesia, pregnant women who used MCHB were more inclined to take advantage of antenatal and postnatal care services (JICA, 2012). In Dhaka, Bangladesh another study showed higher knowledge on MCH issues, better practices in $\mathrm{MCH}$ care, and higher utilization of $\mathrm{MCH}$ services among mothers with the booklets than among those who used alternative health cards (Bhuiyan et. al., 2004). In addition, Nakamura (2010), indicated that the MCHB can change the behavior of mothers during pregnancy and child-rearing. This was evidenced in Bangladesh in a pilot study where $95.2 \%$ of mothers with the booklet received tetanus toxoid vaccine while only $53.3 \%$ of those without received tetanus toxoid vaccine. All these studies have been done outside Kenya. This necessitates a study in Kenya to compare the findings, bringing in new knowledge that can influence policy formulation in Kenya.

A study done in Switzerland showed that of the parents who utilized the booklet, $10 \%$ consulted it many times while $4 \%$ never consulted the information in the booklet (Biel et. al., 2007). In addition, Biel etal., (2007) states that, utilization of the booklet by the mother decreased as the child grew older. Utilization among children below two years was $68 \%$, decreasing to $40.9 \%$ between two to five years. Among mothers with children below two years, $2.4 \%$ never used the booklet while 1.9\% had stopped using it. A survey among guardians who visited the health facility at 18-months for examination of their children showed that the MCHB is highly utilized by guardians in Japan. Ninety-eight percent of them had read the MCHB while $97.8 \%$ of them had made written entries in it. Only $0.9 \%$ of them had lost theirs. Generally, $87.0 \%$ said it was helpful for child bearing and $81.6 \%$ of them said the record of immunization was useful (Nakamura, 2010). In Kenya, a study was conducted in Turkana (a pastoralist community) indicating $59.4 \%-65.8 \%$ utilization of the child growth card by mothers (Situma et al., 2013). This demands for a study that does not target pastoral communities to establish the level of utilization by mothers/ caregivers to guide program implementation.

A report by JICA indicates an enhanced uptake of Maternal Neonatal Child Health (MNCH) services and improved communication between health service providers and their clients while a KAP survey in Palestine revealed an increase in the acquisition of knowledge and better communication between health service providers and clients, and between the clients and their family members (JICA, 2012), yet in Western Kenya, there is scanty information about how MCHB has contributed to $\mathrm{MNCH}$ services in Kenya.

Among health care workers, a study carried out in Switzerland among physicians and pediatricians (71\% private) revealed that $85 \%$ were positive, $9 \%$ were indifferent, and $6 \%$ were negative since they felt that the health booklet was inadequate in documenting child health data. In general pediatrics, $58 \%$ regularly used the booklet while $7 \%$ seldom or never use it. Utilization in general pediatrics among children two to three years was 31\% (Biel et al., 2007). In this particular study, private physicians and pediatricians utilized MCHB as a medical record, an area that needs to be investigated to establish whether in western Kenya physicians and pediatricians appreciate MCHB as a source of medical record information.

\subsection{Study Justification}

Maternal and Child Health care is a major area of focus for a number of countries around the world. Kenya Vision 2030, emphasizes on strengthening communities to become better participants in their own health care through strengthening health centers to provide more of preventive as opposed to curative services. MCHB provides information that empowers mothers and Community Health Workers to make a difference in their own health or that of their child. According to Nakamura (2010), MCHB has the potential, both to improve maternal knowledge and to increase utilization of maternal health services as evidenced in a study in West Sumatra, Indonesia. However, there is scanty information about how MCHB has contributed to MNCH services in Kenya. The study aims to establish the level of awareness, utilization of the MCHB by health workers and mothers. This study will contribute towards achieving the Vision 2030 under the social pillar which works towards ensuring universal access to health care services, the sustainable development goal 3, that looks at good health and well-being for people by ensuring healthy lives and promote well-being for all at all ages and finally achievement of the big 4 agenda that also emphasizes on achieving universal health care.

\subsection{Methods}

The Western Region of Kenya, borders Uganda, and is one of the former Kenya's eight administrative regions. It is to the west of the Eastern Rift Valley and is inhabited mainly by the Luhya people. The region was divided into four study 
strata; Busia County, Kakamega County, Vihiga County and Bungoma Counties, based on administrative boundaries. The study design was a cross sectional descriptive study based on the health of mothers attending MCH in the health facilities. From each stratum, the county referral hospital was purposively selected and other health facilities were randomly selected. Selected health facilities included government owned, Faith Based Organizations (FBO) and private facilities. Proportionate sampling was done and both rural and urban facilities were studied. Purposive sampling was done to determine the area of study, counties to be studied and referral hospitals to be included in the study. Simple random sampling was done to determine the health facilities to be included in the study. Systematic random sampling was used to determine clients to be included in the study. Observation were done to all MCHB. Fischer's formula (1998) was used to establish sample size. The total sample size for the study was 982. The sample was drawn proportionate to the population from four counties.

\subsection{Statistical Analysis}

Quantitative data was collected through; structured questionnaires, key informants guides and Observation Checklist. Data was analyzed using SPSS Version 20.

\section{Results}

\subsection{Demographics}

Total respondents were: Kakamega 246 (25.1\%), Vihiga 253 (25.8\%), Bungoma 244 (24.8\%) and Busia 236 (24\%) making a total of 982 respondents. Overall for age, 3.3\% (32) were less than 20 years, $52.4 \%$ (513) were between 20 to 30 years, $40.4 \%$ (396) were between 30 to 40 years, and 3.9\% were above 40 years. This similar trend was observed within counties with majority between 23 to 40 years: Busia 227 (96.2\%), Bungoma 97.6\% (238), Vihiga 92.1\% (233) and Kakamega 85.8\% (211). Literacy levels were generally high. Overall, 2\% (20) had some level of primary education, 56.7\% (555) had secondary education, and $41.3 \%$ (404) had tertiary education. This similar trend on literacy was also seen among the counties. In Bungoma and Busia, the level of education between tertiary and secondary was similar (51\% and 53\%). However, in Vihiga and Kakamega the level of secondary education was significantly higher at $64 \%$ and $70 \%$ respectively.

Of all the respondents, $75.8 \%$ (742) were married, $15.2 \%$ (149) were single, $6.7 \%$ (66) divorced, $1.4 \%$ (14) separated, and $0.8 \%$ (8) widowed. Within the counties, the number married was 233 (94.7\%) in Kakamega and 234 (92.5\%) in Vihiga. In Bungoma 169 (69.3\%) are married with 18\% and $12 \%$ being single and divorced; Similar to Busia with $45 \%$ (106) married, 36\% (84) single and $13 \%$ (30) divorced.

Overall 47.1\% (461) are in formal employment, 20.4\% (200) are housewives and 31.8\% (311) in informal employment. In Kakamega 54.1 \% (133) are in formal employment, 48.4\% (122) in Vihiga, 42.2\% (103) in Bungoma, 43.6\% (103) in Busia. In Bungoma and Busia, the formal and informal employment are almost similar with Vihiga having the highest number of housewives at $9.8 \%$.

In general, $93.2 \%$ (912) of the clients interviewed delivered in a health facility while the remaining $6.8 \%$ delivered at home. This was mirrored in all the counties; 82.9\% (204), 96\%, 99.2\%, 95 \% delivered in a facility in Kakamega, Vihiga, Bungoma and Busia Counties respectively.

Generally, 27\% often read the MCHB at home while $69.5 \%$ read it sometimes. 3.5\% rarely or never read the MCHB. This trend was almost similar within all the counties. In Kakamega, Vihiga, Bungoma and Busia counties 24\%, 17\%, $29 \%, 39 \%$ read often while $75 \%, 78 \%, 69 \%, 54 \%$ read sometimes.

By and large, both doctors and nurses explained some part of the MCHB to their clients. 78\% (761) of doctors and $13 \%$ (131) of nurses gave explanations to their clients. $68 \%, 83 \%, 88 \%$, and $73 \%$ received information from nurses in Kakamega, Vihiga, Bungoma and Busia respectively; while $6.5 \%, 8 \% .12 \%, 27 \%$ received information from doctors. The others received information from other cadres.

Of all the mothers assessed, 98.7\% (966) received the MCHB at MCH while 1.3\% (13) received it during delivery. The situation was similar within the counties: 98.7\% (233) in Busia, 98.8\% (250) in Vihiga, 97.2\% (239) in Kakamega. It's only in Bungoma where a greater number (100\%) of the mothers received the booklet in $\mathrm{MCH}$; $\mathrm{p}$ value 0.044 .

About forty-six percent (46.3\% - 453) received the MCHB from either the dispensary or health center, $41.6 \%$ (407) from a MOH hospital, 6.5\% (64) from an FBO health center or dispensary and 3.6\% (35) FBO hospital and 1.9\% (19) from a private facility. Half received their MCHBs from MOH dispensaries and health centers in Busia, Vihiga and Kakamega while in Bungoma $42.6 \%$ received it from the $\mathrm{MOH}$ hospital.

Overall, 27\% (266) had 4 ANC visits, 48.6\% (476) 3 ANC visits, 18.8\% (184) 2 visits, $0.9 \%$ (9) one ANC visit, 4.3\% (42) more than four visits. In Busia and Bungoma, the number of women who attended 3 and 4 ANC visits were almost similar (46\% and 43\%: $41 \%$ and $41 \%$ respectively). However, in Kakamega, ANC 3 was at $60.2 \%$ and ANC 2 was at $34 \%$. In Vihiga ANC 3 was $47 \%$ while ANC 2 and 4 was at $21 \%$.

Sixty one percent (597) of the clients always brought their MCHB when visiting the facility, 34.2\% (335) carried it sometimes, while the rest did not carry or remember to. In Busia, Bungoma, Vihiga and Kakamega, those who always carried their MCHB were 61\% (597), 61\% (149) and 65\% (164) respectively. In Kakamega there was no significant difference between those who carried the MCHB always and sometimes.

Overall, 93.2\% (912) of the clients interviewed delivered in a health facility while the remaining $6.8 \%$ delivered at home. This was mirrored in all the counties; 82.9\% (204), 96\%, 99.2\%, 95 \% delivered in a facility in Kakamega, Vihiga, Bungoma and Busia Counties respectively. 


\subsection{Factors Influencing Utilization by Mothers}

$60.5 \%$ of those above 40 attended 3 ANC visits, $43.4 \% 30$ to 40 years attended 3 visits, $58.9 \% 20$ to 30 yeas attended one visit and $46.9 \%$ of those less than 20 years attended 3 visits. Lowest ANC attendance in terms of number of visits was among those 20 to 30 years, $p$ value 0.012 . However, there was no significant difference between place of delivery and age.

3 ANC visits were the highest attended regardless of the level of education. However, 2 ANC visits and more than 4 ANC visits were an average of $12 \%$. Forty eight point six percent visited ANC 3 times while $0.9 \%$ visited it once; $p$ value 0.014.Facility deliveries were higher among tertiary (94\%) than primary level $(75 \%)$ of education. Home deliveries were also significantly higher among primary school (25\%) than tertiary level (6\%); pvalue 0.010 .

\subsection{Utilization by Health Care Workers}

Among the MCHBs observed, for birth plan, 54\% (532) was complete, and $45.6 \%$ (446) had been filled though incomplete. The scenario was similar across Vihiga, Bungoma and Busia with 51\% (128), 45\% (109) and 50\% (119) of the information on the birth plan completely filled. However, the percentage of birth plan completely filled was higher in Kakamega at 72\%. The rest were incompletely filled. In general, 68.8\% (674) of the maternal profiles had been filled while $31.2 \%$ (305) were incomplete. Across the counties, the maternal profiles completely filled were $83.7 \%$ (206), 57.7\% (146), $55.7 \%$ (136), 78.8\% (186) for Bungoma, Kakamega, Vihiga, and Busia Counties respectively. For the section on medical and surgical history, 92.9\% (908) was filled and 7.1\% (69) incompletely filled. This was similar across the counties; $p$ value0.51. For physical examination, 88.5\% (866) was complete while $11.5 \%$ (113) was incomplete. Across counties, 95.9\% (236), 93.3\% (236), 79.5\% (194), and 84.7\% (200) were completely filled in Kakamega, Vihiga, Bungoma and Busia counties respectively. Bungoma and Busia were the counties with the greatest number where physical examination was incomplete at $20.5 \%$ (50) and $15.3 \%$ (36) respectively. For infant feeding, $75.8 \%$ (742) were filled with $23 \%$ (225) incompletely filled and 1.2\% blank. In Busia 76.6\% (181), Bungoma 84.8\% (207), Kakamega 68.8\% (174), 73.2\% (180) in Vihiga were completely filled on infant feeding. These differences between sub counties were significant ( $p$ value 0.004). Eight six point five percent (847) of the pages on ANC profile was filled, $13.4 \%$ (131) incomplete and $0.1 \%$ (1) not filled at all. Kakamega had the greatest number of incompletely filled ANC profile at 25.6\% (63) and the lowest completely filled pages at $74 \%$ (182).

For the status of present pregnancy $84 \%$ (822) was complete with 16\% (157) incomplete. In counties $83.5 \%$ (197), 84\% (205), 83.4\% (211), 85\% (209), were complete in Busia, Bungoma, Vihiga and Kakamega respectively. These were not significant; p value 0.961 . For the chart on weight gain, $76.5 \%$ (749) were complete, $23 \%$ (225) incomplete and $0.5 \%$ (5) not filled at all. It is only in Busia where the weight gain chart was not filled. This was significant $p$ value 0.001 . For preventive services, $72.5 \%$ (710) was complete, $27.2 \%$ (266) was incomplete and $0.3 \%$ (3) not filled at all. This was mirrored in all the counties p value 0.165 . For delivery $90 \%$ (888) was filled, $9.1 \%$ (89) were incomplete and $0.1 \%$ (1) was not filled. In Bungoma, those with incomplete information on delivery were 15.2\% (37). For post-natal examination (mother), 85.7\% (839) was complete, 14.2\% (139) was incomplete and 0.15 (1) was not filled at all. Busia was $91.9 \%$ (839), Bungoma 85.2\% (208), Vihiga 82.2\% (208) and Kakamega 83.7\% (206); pvalue 0.015. Under post-natal examination on the section for the baby, $85 \%$ (832) was complete, $14.9 \%$ (146) was incomplete, and $0.1 \%$ (1) not filled. The situation was similar across the counties and was not statistically significant. For cancer screening, 19.3\% (189) was complete, $80.2 \%$ (785) incomplete and $0.5 \%$ (5) not filled. Completeness varied among counties: Kakamega 25.2\% (62), Vihiga 23.3\% (59), Bungoma 18.9\% (46) and Busia 19.3\% (22). The five whose pages had not been filled at all were from Bungoma County. Under family planning, 50.2\% (491) was complete, $47.9 \%$ (469) incomplete, and 1.9\% (19) not filled at all. Kakamega, Vihiga and Bungoma was almost similar for completeness at 52\%, 57.3\%, and 50.8\% respectively. Busia had the lowest level of completeness at 39.8\%.The differences among the counties was significant; $\mathrm{p}$ value 0.005 . For immunization 95.5\% (935) was complete, and 4.5\% (44) incomplete. Among the counties completeness was between 91.5\% (216) in Busia and 98.8\% (250) in Vihigap value 0.001.

Under health worker consultation, 62.7\% (614) was blank/ never filled at all. Among counties, 62.6\% (154), 48.6\% (123), $64.8 \% 7$ (158), 75.8\% (179) had not been filled in Kakamega, Vihiga, Bungoma and Busia respectively.

Birth plan, overall was $54.3 \%$ completeness. The highest completeness was in Kakamega at $87.9 \%$ while the lowest was in Busia at 32.4\%. For maternal profile, overall, it was 68.8\% (674) complete. Kakamega had the highest among all facilities at hospitals $84 \%$ and health centers $87.5 \%$ respectively. For dispensaries, the highest was Busia at $93.7 \%$ (59). The lowest were Bungoma hospitals at 58.5\% (55), Bungoma health centers at 34.5\% (30), dispensary was 56.8\% 50 Vihiga dispensaries. The level of facility influenced the filling of the medical surgical history; $p$ value 0.019 and the filling of pages of infant feeding as the dispensaries were four times more likely not to have filled the pages on infant feeding compared to the other facilities; $p$ value 0.025 . Dispensaries were 1.1 times more likely than hospitals to have the weight gain chart filled; $\mathrm{p}$ value 0.003 . The number of ANC visits significantly affected the filling of the page on maternal profile, the higher the number of visits, the higher the completeness. Those who delivered in a facility were more likely to have information on family planning captured in their MCHB than those who delivered at home p value 0.003 .

\subsection{Factors Affecting Utilization By Mothers}

The age of the mothers was found to influence the number of ANC visits with highest number of ANC visits among the older cohort; $p$ value 0.012 . Overall, $93 \%$ delivered in a health facility with $6.8 \%$ home deliveries. Level of education influenced place of delivery as higher education was associated with facility delivery; $p$ value 0.010 . 


\section{Discussion}

The MCHB is a complete booklet. Initially, mothers used to have a separate card that was blue in color that was used only during pregnancy. When the child was born, a yellow card called 'road to health' was used from birth until the child reached five years. However, the MCHB is holistic since it covers the entire period from pregnancy until the child is five years old. Those visiting the health facility are more likely to have information captured on family planning; $p$ value 0.003. This is because given the emphasis on family planning by the ministry of health, those in the facility receive the counselling and are offered methods based on their choices.

Worldwide only $70 \%$ of women ever receive any ANC, whereas in industrialized countries more than $95 \%$ of pregnant women receive ANC (WHO, 2003). Overall, 98\% of mothers received their MCHB from the MCH as opposed to 1.3 $\%$ who received it during delivery. This reflects overall ANC attendance. The $1.3 \%$ who received during delivery never attended ANC. This is similar to the 95.5\% ANC attendance and 3.9\% who never attended any ANC (KDHS, 2014). Most mothers in the study received the MCHB during the antenatal clinic in comparison to the Gaza strip where $97 \%$ received during that period and $11 \%$ during delivery (Kibaru \& Otara 2015) this is comparable with the survey in West Bank where only 46\% received the booklet during antenatal and 50\% during delivery (Imamura Kenshiro, 2012). Gisele et al., 2014 noted that unavailability of the MCHB and mothers forgetting to carry it were challenges.

Regular antenatal care is helpful in identifying and preventing adverse pregnancy outcomes when it is sought early in the pregnancy and is continued until delivery (KDHS, 2014). The World Health Organization recommends that women have at least four antenatal care visits during each pregnancy (WHO, 2007a). In Kenya, 3.3\% had one visits, 34.8\% 2 to 3 visits, $57.6 \%$ more than four visits. In the western Kenya where our study was conducted this was $4 \%, 41.8 \%$ and $51.3 \%$ for one, 2 - 3 and more than 4 ANC visits respectively (KDHS, 2014). Maternal and peri-natal deaths occur among mothers who did not attend ANC (WHO, 2007b). These findings are different from those of our study where generally ANC 2 and 3 were highest at $67.4 \%$, and more than 4 ANC visits at $31.3 \%$ and $5 \%$ one visit and the high figures in our study were attributed to the fact that this was not a survey unlike KDHS but data was collected among mothers who were in the facility for various services.

The filling of the various MCHB pages demonstrates the importance health workers attribute each section with immunization being the most complete and this trend was similar in all health facilities. Kitenge et.al, (2013) however attributes prioritization of immunizations to the health workers' attachment to the importance of immunizations in reducing morbidity and mortality. A study in Gaza found the level of completeness as follows: personal information 99\%, history 95\%, ANC follow up 97\%, hospital examination 39\%, postnatal examination 57\%, newborn assessment $64 \%$, immunization 97\%, growth monitoring 98\% (Imamura, 2012).This similar scenario as described by Kitenge et al.,2013 ,can be applied to Kenya situation where the most common filled section of the MCHB is on vaccinations, vitamin A supplementation and deworming (Kibaru and Otara, 2015; Kitenge et.al., 2013).

Antenatal care (ANC) from a skilled provider is important to monitor pregnancy and reduce the risk of morbidity for mother and baby during pregnancy and delivery (KDHS, 2014). A number of studies have shown that there is knowledge gap among health workers on the MCHB (Gisele, 2014: Kibaru \& Otara, 2015). This impacts the information imparted to the clients.

Incomplete filling of the MCHB was attributed to health worker knowledge, heavy workload and loss of the MCHB or forgetting it at home by caregivers and mothers (Kibaru and Otara, 2015; Opondo, 2015; Kitenge et.al, 2013). Overworked clinic workers may omit weight plotting (Tarwa \& Villiers, 2007). Despite these challenges, Gisele et al (2014) notes that the MCHB can contribute to changes in child care if professionals, mothers and families apprehend its meanings. In western province home deliveries were at $51.3 \%$ verses facility delivery of $47 \%$. However, at national level $37 \%$ were home deliveries versus $61 \%$ facility deliveries (KDHS, 2014).The disparity is attributed to KDHS being a survey, while this study collected data purposively from women of reproductive age visiting ANC clinic.

\subsection{Factors Affecting Utilization}

Among those aged forty years and above, $60.5 \%$ attended 3 ANC visits while $47 \%$ of those less than twenty years attended one visit. The number of ANC visits was influenced by the age of the clients'; $p$ value0.012. The older clients tended to have more number of ANC visits compared to the younger age cohorts.

The level of education determined place of delivery. Facility deliveries were significantly higher among tertiary (94\%) and secondary school (93\%) attendants and home deliveries highest among primary school leavers (25\%); $\mathrm{p}$ value 0.014. Kibaru \& Otara (2015) found that the level of education, number of deliveries and care provider explaining the content of MCHB were statistically significant determinant of the likelihood that the mother would read the booklet.

Of the mothers, $99.6 \%$ attended ANC and $98.6 \%$ of them were given the standard MCHB. However, only $59 \%$ of them were explained to the contents of the book by the health care providers and up to $33.5 \%$ of the mothers did not read the instructions in the $\mathrm{MCHB}$

According to Imamura (2012), 64\% of the WRA had read all of it, $28 \%$ part of it and $8 \%$ never. The parts of the MCHB found most helpful were: child development $49 \%$, child nutrition $47 \%$, breastfeeding $41 \%$ and guide for pregnant women $39 \%$. All mothers should be given the knowledge on maternal and child health as early as antenatal clinic. Increased knowledge by parents increases demand for services (Musoke, 2016). Since most mothers accompany their children to the hospital, this is a good opportunity to educate mothers (Tarwa \& Villiers, 2007). The major challenge faced is the lack of sensitization of mothers by the health care workers on its importance (Gisele et al., 2014) while Tarwa \& Villiers, (2007) noted that health workers do not ask for the MCHB at the consultation resulting in missed opportunities 
and was also underutilized for child monitoring. Imamura (2012), further reports that women who received the MCHB during ANC and brought it along during visits were 96\%, reading the MCHBs: 93\% of women read the health education/information pages of the MCHB, communicating with Health Care Providers: $71 \%$ of women were given an explanation about the MCHB by health care providers and communicating with Family: $65 \%$ of women had shown their MCHHB to their family members.

One study attributes the challenges with the MCHB utilization to staff shortages, inadequate equipment and commodities, and poor attendance of caregivers for sessions resulting in missed opportunities (Kitenge et.al, 2013). For children under five, growth monitoring is a universal pillar (Garner et. al, 2000). Training of health workers on plotting of accurate weights, identifying those who need interventions and reducing missed opportunities is therefore key (Tarwa \& Villiers, 2007). A study by Sistiarani et al., 2016 demonstrated an increase in utilization by $31 \%$ and skills by $23 \%$ after health workers were trained.

\section{Conclusion}

The success of the MCHB is therefore dependent on the knowledge, dedication and cooperation of both mothers/ caregivers and health workers (Tarwa \& Villiers (2007)). Involving mothers and ensuring they understand has enabled increased utilization of growth monitoring services (Wilkinson \& Morley 1998 \& Brannstrom et.al., 1998). Therefore, health workers should educate the mothers on all its aspects also remind mothers to always have the MCHB whenever they visit the facility (Tarwa \& Villiers, 2007).

JICA (2012) reports enhanced uptake of MNCH services and improved communication between health service providers and their clients while a KAP survey in Palestine revealed an increase in the acquisition of knowledge and better communication between health service providers and clients, which is indispensable to achieving a continuum of care.

\section{References}

i. Aden AS, Brannstrom I, Mohamud KA, Persson LA, Wall S. The growth card - a road to health card? Maternal comprehension of the growth monitoring cards in two Somali villages. Paediatr Perinat Epidemiol 1990;4(3):340-50.

ii. Asuzu MC. A comparative study of the commonly used nutritional assessment tools for primary health care. East Afr Med J 1991;68(11):913-22.14

iii. Bhuiyan S. U., Nakamura Y., Qureshi N. A. (2004). Study on the Development and Assessment of Maternal and Child Health (MCH) Handbook in Bangladesh. Journal of Public Health and Development 2006 Vol. 4 No. 2 p 45-60

iv. Biel R.C., Chêne-Bourg N. P., Luzern H. H., Grenchen T. B., Biel C. M., (2007). Evaluation of the Swiss child health booklet. PEDIATRICA Vol 18. No. 52007 pp. 49 - 51

v. College of health sciences. Utilization of the Mother-Child Health Booklet by Healthcare Workers and Caregivers at Mbagathi District Hospital. -College of Health Sciences (CHS) 2015. [2656] http:/ / hdl.handle.net/ 11295/ 94950

vi. Colti Sistiarani, Arif Kurniawan, Endo Dardjito. Impact of Health Volunteer Training to Improve Utilization of Maternal Child Health (MCH) Book in Kalibagor Indonesia. International Journal of Public Health and Clinical Sciences. 2016 E-ISSN: 2289-7577. Vol. 3:No. 3

vii. Division of Nutrition. Maternal, Infant and Young Child Nutrition: National operational guidelines for health workers. Ministry of Health, Kenya2013

viii. Fagbule D.O., Olaosebika A., Parakoyi D.B. Community Awareness and Utilization of Growth Charts in Semi Urban Nigeria Community. East African Medical Journal. 1990. Vol. 67 (2): 69-74

ix. Fleischman Janet (2011). Lessons from Kenya for the Global Health Initiative. A report of the CSIS global health policy center.

x. G Kitenge, \& I Govender. Nurses' monitoring of the Road to Health Chart at primary healthcare level in Makhado, Limpopo province, South African Family Practice, 2014. 55:3, 275-280, DOI:10.1080/ 20786204.2013.10874350

xi. Gisele Nepomuceno de Andrade, Tércia Maria Ribeiro Lima Rezende, Anézia Moreira Faria Madeira (2014). Child Health Booklet: experiences of professionals in primary health care Rev. esc. enferm. USP vol.48 no.5 São Paulo Oct. 2014

xii. Health Management Information System. Health Sector Indicator and Standard Operating Procedures Manual for Health Workers. 2008.Imamura Kenshiro (2012). Overview of the MCHHB in Palestine. Ministry of Health

xiv. Imamura Kenshiro (2012). Overview of the Mother and Child Health Handbook (MCHHB) in Palestine. Together Toward a Better Life Style for the Palestinian People. October 2012.

xv. Isaranurug S. Maternal and Child Health Handbook in Thailand. Journal of International Health. 2009; 24(2):61-66.

xvi. JICA. Every Life is precious. Maternal New born and Child Health. 2012.

xvii. Kenshiro Imamura. Overview of MCHHB in Palestine. Towards a better lifestyle for the Palestinian people. 2012

xviii. Kibaru E. Gathoni, Otara A. Magembe. Utilization of Mother and Child Booklet among Mothers Attending Well Baby Clinic in Nakuru Central District. International Journal of Science and Research (IJSR).2015 Vol. 4 Issue 4 ISSN (Online): 2319-7064. Pg 2642 - 2648

xix. Nakamura Yasuhide. Maternal and Child Health Handbook in Japan. JMAJ 2010. 53(4): 259-265.

xx. Opondo, McogaaPaul Garner, Ratana Panpanich, Stuart Logan. (2015). Is routine growth monitoring effective? A systematic review of trials. Arch Dis Child 2000; 82:197-201 197

xxii. Rachel N Musoke.The Long Road to Newborn Survival: Working with Parents, Families and Communities to Reduce Preventable Neonatal Deaths Pediatrics \& Neonatal Biology Open Access Pediatr Neonat biol 2016, 1(1): 000105.

xxiii. Sohal H, Wilkinson D, Morley D. The growth monitoring teaching aid rapidly improves mother's understanding of growth curves. Trop Doct 1998; 28(3):160-2. 
xxiv. Tarwa C, andDe Villiers FPR. The use of the Road to Health Card in monitoring child health. SA Fam Pract 2007;49(1):15

xxv. Wambui S. Kenya mover to curb child mortality. Capital News [Internet]. 2010 Apr 23 [cited 2011 Mar 18]. http:/ / www.capitalfm.co.ke/ news/ Kenyanews/ Kenyamoves-to-curb-child-mortality-8222.html

xxvi. World Health Organization (2007). Integrated Management of Pregnancy and Childbirth: Standards for Maternal and Neonatal care

xxvii. World Health Organization. Antenatal care in developing countries. Promises, achievements and missed opportunities. An analysis of trends, levels and differentials, 1990-2001. Geneva, World Health Organization, 2003. 